\title{
EFEITO DA INTERAÇ̃̃O DA APLICAÇÃO DE CLORETO DE MEPIQUAT COM ABAMECTIN SOBRE A POPULAÇÃO DO ÁCARO RAJADO E PRODUÇÃO DE ALGODÃO EM CAROÇO
}

José Roberto Scarpellini ${ }^{1}$

\section{RESUMO}

Com o objetivo de avaliar o efeito vacina sobre o ácaro rajado Tetranychus urticae em algodoeiro cv. deltaopal utilizando Cloreto de Mepiquat em mistura com Abamectin, foi realizado este ensaio em Ribeirão Preto, SP, de 08/12/2000 a 11/05/2001. Aos 30, 38 e 60 DAE (dias após a emergência) foram infestadas todas as parcelas com colônias de ácaros, distribuidas aleatoriamente (100 folhas infestadas). Aos 44, 56 e $68 \mathrm{DAE}$ procedeu-se às pulverizações, utilizando os tratamentos: 1 - abamectin $18 \mathrm{CE}+$ cloreto de mepiquat $(2 \mathrm{x}$ de $5,4 \mathrm{~g}$ i.a.I ha $+3 \mathrm{x}$ de $15 \mathrm{~g}$ i.a./ha); 2 - abamectin $18 \mathrm{CE}+$ cloreto de mepiquat (3 $x$ de 3,6 g i.a./ha $+2 x$ de 15 g i.a./ha); 3 - abamectin $18 \mathrm{CE}+$ cloreto de mepiquat $(10,8 \mathrm{~g}$ i.a./ha $+3 \mathrm{x}$ de $15 \mathrm{~g}$ i.a./ha); 4- abamectin $18 \mathrm{CE}$ (10, 8 g i.a./ha); 5-Vertimec $18 \mathrm{CE}$ ( 2 x de 5,4 g i.a./ha); 6 - abamectin $18 \mathrm{CE}(3 \mathrm{x}$ de $3,6 \mathrm{~g}$ i.a./ha); 7 - cloreto de mepiquat $(3 \mathrm{x}$ de $15 \mathrm{~g}$ i.a./ ha); 8- diafenthiuron 500 PM a 400 g i.a./ha, 9- Testemunha. Nas aplicações parceladas de abamectin em 3 vezes de $3,6 \mathrm{~g}$ i.a./ha ou 2 vezes de $5,4 \mathrm{~g}$ i.a./ha (efeito vacina), adicionado ao cloreto de mepiquat a $15 \mathrm{~g}$ i.a./ha em 3 vezes, o ácaro rajado não se desenvolveu e a produção de algodão em caroço foi aumentada, mas sem comprovação estatística.

Palavras chave: acarologia, fỉsiologia, controle químico, produtividade.

1 Polo Regional de Desenvolvimento Tecnológico do Centro Leste, APTA E-mail:jrscarpellini@biologico.br C.P. 271 CEP 14001-970 - Ribeirão Preto, SP. 


\section{ABSTRACT}

\section{INTERACTION EFFECT OF MEPIQUAT CHLORIDE WITH ABAMECTIN ON THE POPULATION OF TWOSPOTTED MITE AND SEED COTTON PRODUCTION}

The interaction effect of mepiquat chloride with abamectin on the population of twospotted mite Tetranychus urticae and seed cotton production were evaluated in the present experiment, conducted at the Experimental Station of Ribeirão Preto, from December/2000 to May/ 2001. On 30, 38 e 60 DAE (days after emergence) all plots received 100 infested leafs with mites colony. On 44, 56 e 68 DAE the applications were made, using the folowing treatments: 1 - abamectin $18 \mathrm{CE}+$ cloreto de mepiquat ( $2 \mathrm{x}$ at the $5,4 \mathrm{~g}$ i.a./ha $+3 \mathrm{x}$ at the $15 \mathrm{~g}$ i.a./ha); 2 - abamectin $18 \mathrm{CE}+$ cloreto de mepiquat ( $3 \mathrm{x}$ at the $3,6 \mathrm{~g}$ i.a. $/ \mathrm{ha}+2 \mathrm{x}$ at the $15 \mathrm{~g}$ i.a./ ha); 3 - abamectin $18 \mathrm{CE}+$ cloreto de mepiquat $(10,8 \mathrm{~g}$ i.a./ha $+3 \mathrm{x}$ at the 15 g i.a./ha); 4- abamectin $18 \mathrm{CE}$ (10, 8 g i.a./ha); 5- Vertimec $18 \mathrm{CE}$ (2 $\mathrm{x}$ at the 5,4 g i.a./ha); 6- abamectin $18 \mathrm{CE}(3 \mathrm{x}$ at the $3,6 \mathrm{~g}$ i.a./ha); $7-$ cloreto de mepiquat ( $3 \mathrm{x}$ at the $15 \mathrm{~g}$ i.a./ha); 8- diafenthiuron $500 \mathrm{PM}$ (400 g i.a./ha) and 9- Check. Results showed that mites did not develope with abamectin $18 \mathrm{CE}$ with 3 applications (3,6 g i.a./ha) or twice $(5,4 \mathrm{~g}$ i.a./ha) plus mepiquat chloride (vacin effect); the seed cotton production was increased, but not supported by statistical analysis.

Key words: acarology, fisiology, chemical control, yield.

\section{INTRODUÇÃO}

O Estado de São Paulo possui área plantada com algodão ao redor de 67.517 ha (Caser et al., 2001). Há vários fatores responsáveis pela produção aquém do potencial do algodoeiro, entre os quais a falta de monitoramento e controle no momento adequado das pragas e doenças que a infestam, constituindo os ácaros um grave problema, principalmente pela ocorrência constante, em determinadas épocas do ano, em níveis populacionais muito altos (Nakano et al., 1981). 
O ácaro rajado Tetranychus urticae (Koch, 1836) (Acari: Tetranychidae) é problema constante na cultura algodoeira. É um artrópodo de coloração esverdeada com manchas dorsais escuras; mede cerca de $0,46 \mathrm{~mm}$ (fêmeas) e $0,25 \mathrm{~mm}$ (machos), os ovos são esféricos e de tonalidade amarelada, são postos entre fios de teia que o ácaro tece na página inferior das folhas (Gallo et al., 1988). Segundo Chiavegato (1971), a população do ácaro está relacionada com a idade das plantas, ocorrendo normalmente a partir dos 60 e 70 dias após a germinação. Oliveira (1971) obteve na região de Jaboticabal-SP, uma redução quantitativa expressa em peso de algodão em caroço, da ordem de 7,8 a 25,5\%, de acordo com os diversos níveis de infestação constatados. Reis (1972) obteve variação de 5,39 a 30,0\%, também dependendo da infestação encontrada. Ambos concluíram que a qualidade da fibra e da semente ficam significativamente prejudicados.

É frequente a utilização de cloreto de mepiquat (Pix) na lavoura algodoeira, com a dose indicada subdividida em duas ou três vezes, sendo esta última com melhores resultados (Lamas et al., 1999). Prasad et al. (2000) afirmam que o uso do cloreto de mepiquat, um regulador de crescimento de plantas, diminui o crescimento vegetativo, reduzindo a altura das plantas. $\mathrm{O}$ peso das maçãs, produção de algodão em caroço e comprimento das fibras é significativamente maior com o uso deste produto. Flint et al. (1996) observaram que o uso do cloreto de mepiquat tem efeito adverso sobre a população de mosca branca Bemisia argentifolii em algodoeiro, em casa de vegetação, reduzindo significativamente o número de adultos, ovos e ninfas da praga, nas parcelas tratadas com o produto. Observações pessoais do autor (safra 1999/ 2000 ) indicaram que abamectin (Vertimec $18 \mathrm{CE}$ ) a $10,8 \mathrm{~g}$ i.a./ha aplicado em mistura ao cloreto de mepiquat (Pix) a $25 \mathrm{~g}$ i.a./ha, apresentou menor tamanho de plantas, tonalidade verde mais escura, melhor efeito residual sobre ácaro rajado T. urticae e produção de algodão em caroço maior que aplicado em separado.

O objetivo deste trabalho é estudar o efeito vacina utilizando Cloreto de Mepiquat (Pix), em mistura com Abamectin (Vertimec 18 CE) sobre o ácaro rajado Tetranychus urticae em algodoeiro cv. deltaopal. 


\section{MATERIAL E MÉTODOS}

O experimento foi instalado em condições de campo, na Estação Experimental do Instituto de Zootecnia em Ribeirão Preto, SP, na safra agrícola (2000/2001), com o cultivar Deltaopal, tratado com thiamethoxam (Cruiser 700 WS a 300 g p.c. $/ 100 \mathrm{~kg}$ ). Usaram-se 4 blocos ao acaso, com 9 tratamentos. Cada parcela constou de 8 linhas de $10 \mathrm{~m}$ de comprimento, com uma bordadura de $1 \mathrm{~m}$ entre blocos e $1 \mathrm{~m}$ entre parcelas. As pulverizações foram realizadas aos 44,56 e $68 \mathrm{DAE}$, conforme a escala de aplicação do tratamento, com pulverizador costal $\mathrm{CO} 2$, a pressão constante de $40 \mathrm{lb} / \mathrm{pol}^{2}$ e bico X3 com volume de calda de $200 \mathrm{~L} / \mathrm{ha}$. Anotaram-se horários de aplicações, umidade relativa e temperatura. Os tratamentos e dosagens utilizadas foram os constantes da Tabela 1.

Tabela 1. Tratamentos empregados no experimento. Ribeirão Preto, SP, $08 / 12 / 2000$ a $11 / 05 / 2001$.

\begin{tabular}{|c|c|c|c|c|c|c|}
\hline \multirow[b]{2}{*}{ Tratamentos } & \multirow[b]{2}{*}{ Ingredientes ativos } & \multicolumn{2}{|c|}{ dosagens } & \multicolumn{3}{|c|}{ Aplicaçöes } \\
\hline & & g i.a./ha & mL p.c./ha & 44 DAE & 56 DAE & 68DAE \\
\hline 1- Vertimec + Pix & Abamectin + Cloreto mepiquat & $5.4+15$ & $0,3+0,3$ & $x+x$ & $X+x$ & $x$ \\
\hline 2. Vertimec + Pix & Abamectin + Cloreto mepiquat & $3,6+15$ & $0.2+0.3$ & $x+x$ & $x+x$ & $x+x$ \\
\hline 3- Vertimec + Pix & Abamectin + Cloreto mepiquat & $10,8+15$ & $0,6+0,3$ & $x+x$ & $\mathrm{X}$ & $\mathrm{x}$ \\
\hline 4- Vertimec $18 \mathrm{CE}$ & Abamectin & 10,8 & 0,6 & $x$ & & \\
\hline 5- V'ertimec $18 \mathrm{CE}$ & Abamectin & 5,4 & 0,3 & $x$ & $x$ & \\
\hline 6- Vertimec $18 \mathrm{CE}$ & Abamectin & 3,6 & 0,2 & $x$ & $x$ & $x$ \\
\hline 7-Pix & Cloreto de mepiquat & 15 & 0.3 & $x$ & $x$ & $x$ \\
\hline 8- Polo $500 \mathrm{PM}$ & Diafenthiruon & 400 & 0.8 & & $x$ & \\
\hline 9- Testemunha & $-\cdots$ & ........ & $\ldots .$. & $\ldots$. & $\ldots$ & ..... \\
\hline
\end{tabular}

$\mathrm{DAE}=$ dias após a emergência das plântulas

Aos 30, 38 e 60 DAE (dias após a emergência) foram infestadas todas as parcelas com colônias de ácaros, distribuindo-se aleatóriamente 100 folhas infestadas. As avaliações da população do ácaro rajado foram feitas mediante a contagem de ácaros $\left(1 \mathrm{~cm}^{2}\right)$ na área de maior concentração da folha, em 25 folhas por parcela em 01/02; 06/02; 11/02; 16/02; 23/02; 01/03 e 12/03/2001.

A altura de 25 plantas por parcela foram tomadas aos 95 e 120 DAG, bem como a produção final de algodão em caroço aos 130 e 145 
DAG, nas seis fileiras centrais, desprezadas as bordaduras ( $1 \mathrm{~m}$ de cada lado da parcela).

\section{RESULTADOS E DISCUSSÃO}

Os resultados obtidos estão nas Tabelas 2 e 3, observando-se boa infestação do ácaro rajado na avaliação prévia (Tabela 2), indicando que a infestação do ácaro rajado realizada anteriormente nas parcelas foi positiva.

Tabela 2. Número de ácaros rajados Tetranychus urticae presentes em 100 folhas por tratamento nas avaliações realizadas previamente e aos 5 e 11 dias após a $1^{\mathrm{a}}$ aplicação (DA1A), 4 e 11 após a $2^{\mathrm{a}}$ aplicação (DA2A) e 5 e 15 dias após a $3^{\mathrm{a}}$ aplicação (DA3A). Ribeirão Preto, SP, 08/12/2000 a 11/05/2001.

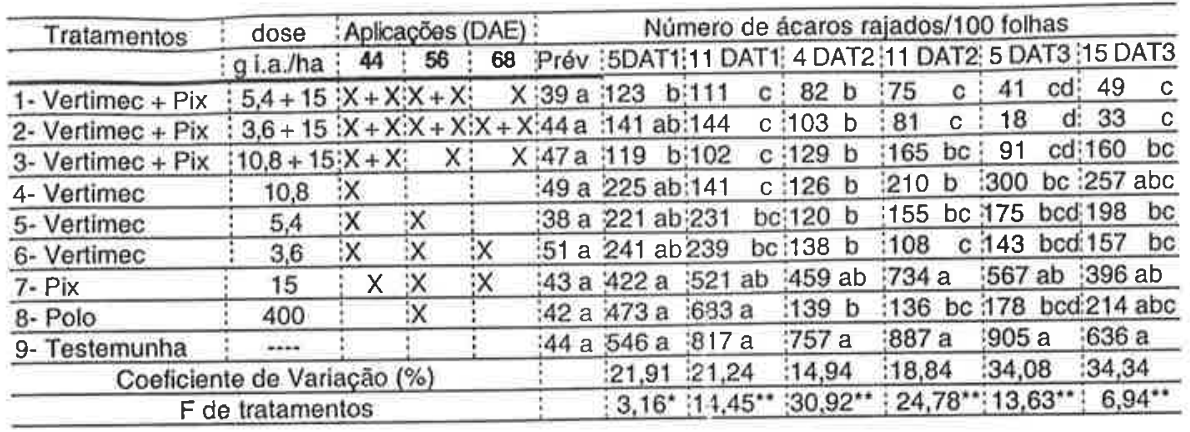

1.Dados transformados em $\sqrt{x+o, 5}$.

2. Médias seguidas por letras minúsculas iguais, na horizontal, não diferem entre si significativamente

Aos 5 dias após a primeira aplicação (DAT1) observou-se redução significativa na população de ácaros, apenas nos tratamentos 1 e 3 , onde utilizou-se abamectin a 5,4 e 10,8 g i.a/ha + cloreto de mepiquat a $15 \mathrm{~g}$ i.a /ha. Os demais tratamentos também reduziram a população de ácaros, com exceção do diafenthiuron $500 \mathrm{PM}$ a $400 \mathrm{~g}$ i.a./ha, que foi aplicado somente na segunda aplicação (maior infestação). 
Tabela 3. Altura de plantas (média de 100 plantas) aos 95 e 120 dias após a emergência (DAE) e produção de algodão em caroço (kg/ha) obtida nos tratamentos. Ribeirão Preto, SP, 08/12/2000 a $11 / 05 / 2001$.

\begin{tabular}{|c|c|c|c|c|c|c|c|c|c|}
\hline \multirow{2}{*}{ Tratamentos } & \multirow{2}{*}{$\begin{array}{c}\text { dose } \\
\text { gi.a./ha }\end{array}$} & \multicolumn{3}{|c|}{ Aplicações } & \multicolumn{2}{|c|}{ Altura de plantas $(\mathrm{cm})$} & \multicolumn{3}{|c|}{ kg algodāo em caroço } \\
\hline & & $01 / \mathrm{Fev}$ & 12/Fev & 24/Fev & $95 \mathrm{DAE}$ & $120 \mathrm{DAE}$ & $\mathrm{kg} / \mathrm{trat}$ & $\mathrm{kg} / \mathrm{ha}$ & $\%$ Acresc, \\
\hline 1-Vertimec + Pix & $5,4+15$ & $x+x$ & $x+x$ & $x$ & 95 & 98 & 9,7 a & 2694 & 18,3 \\
\hline 2- Vertimec + Pix & $3,6+15$ & $x+x$ & $x+x$ & $x+x$ & 94 & 97 & 9,9 a & 2750 & 20,7 \\
\hline 3- Vertimec + Pix & $10,8+15$ & $x+x$ & $\mathrm{x}$ & $\mathrm{X}$ & $100 \quad c$ & $103 \mathrm{c}$ & $9,5 \mathrm{a}$ & 2639 & 15,9 \\
\hline 4- Vertimec & 10,8 & $x$ & & & $118 \mathrm{ab}$ & $122 a b$ & 8.9 a & 2472 & 8, \\
\hline 5- Vert & & $x$ & $x$ & & $115 a b$ & $118 \mathrm{ab}$ & 9,4 a & 2611 & \\
\hline 6- Verti & & $x$ & $x$ & $x$ & $112 \mathrm{~b}$ & $115 \mathrm{~b}$ & 9,6 a & 2667 & 17,1 \\
\hline 7-Pix & 1 & $x$ & $x$ & $x$ & $102 \quad c$ & $104 \quad c$ & 8,8 a & 2444 & 7,3 \\
\hline 8- Polo & 400 & & $x$ & & $125 \mathrm{a}$ & 128 a & $9,6 \mathrm{a}$ & 2667 & 17,1 \\
\hline 9- Testemunha & $\cdots+$. & & & & $126 \mathrm{a}$ & $119 a$ & 8,2 a & 2167 & \\
\hline \multicolumn{5}{|c|}{ Coeficiente de Variacão (\%) } & 1,40 & $\frac{1,16}{1,70 x+1}$ & \multirow{2}{*}{\multicolumn{3}{|c|}{8,44}} \\
\hline \multicolumn{5}{|c|}{$\mathrm{F}$ de tratamentos } & $31,12^{\star \star}$ & $30,78^{\star \star}$ & \multicolumn{2}{|r|}{-1} & \\
\hline
\end{tabular}

1.Dados transformados em $\sqrt{x+o, 5}$.

2. Médias seguidas por letras minúsculas iguais, na horizontal, não diferem entre si significativamente

\% Acrésc $=$ Porcentagem de acréscimo na produção de algodão em caroço, em relação à testemunha

Aos 11 DAT1 todos os tratamentos contendo abamectin a 3,6; 5,4 e $10,8 \mathrm{~g}$ i.a/ha + cloreto de mepiquat a $15 \mathrm{~g}$ i.a /ha e abamectin a $10,8 \mathrm{~g}$ i.a./ha aplicado isolado apresentaram controle eficiente e diferenciaramse dos demais tratament Js. Aos 4 DAT2, com exceção do cloreto de mepiquat a $15 \mathrm{~g}$ i.a./ha, todos os tratamentos diferenciaram-se da testemunha e apresentaram eficiência de controle satisfatória. Aos 11 DAT2: foram obtidos resultados semelhantes, tendo todos os tratamentos diferido do cloreto de mepiquat a $15 \mathrm{~g}$ i.a./ha e testemunha, destacando-se o abamectin em 3 vezes de 3,6 g i.a/ha ou 2 vezes de 5,4 g i.a/ha (efeito vacina), adicionado ao cloreto de mepiquat a $15 \mathrm{~g}$ i.a./ha em 3 vezes.

$\mathrm{Na}$ avaliação realizada aos 5 DAT3 verificou-se que apenas o cloreto de mepiquat a $15 \mathrm{~g}$ i.a./ha em 3 vezes e o abamectin a $10,8 \mathrm{~g}$ i.a./ha aplicado em dose única e isolado não apresentaram eficiência de controle satisfatória, destacando-se o abamectin em 3 vezes de 3,6 g i.a/ha ou 2 


\section{CLORETO DE MEPIQUAT COM ABAMECTIN EM ÁCARO RAJADO 119}

vezes de $5,4 \mathrm{~g}$ i.a/ha (efeito vacina), adicionado ao cloreto de mepiquat a 15 g i.a./ha em 3 vezes.

Resultados semelhantes foram obtidos aos 15 DAT3, quando também o abamectin a $10,8 \mathrm{~g}$ i.a./ha aplicado em dose única e isolado não diferenciou-se da testemunha ou do Cloreto de mepiquat a $15 \mathrm{~g}$ i.a./ha em 3 vezes. O abamectin em 3 vezes de $3,6 \mathrm{~g}$ i.a/ha ou 2 vezes de $5,4 \mathrm{~g}$ i.a/ha (efeito vacina) adicionado ao cloreto de mepiquat a $15 \mathrm{~g}$ i.a./ha em $3 \mathrm{x}$ ainda proporcionava excelente eficiência de controle aos 15 DAT3, indicando o efeito sinérgico destes produtos em aplicações com dose fracionada, funcionando como vacina.

Gavioli et al. (1987) obtiveram resultados satisfatórios no controle do ácaro rajado $T$. urticae, de ocorrência natural, com abamectin a 5 e $10 \mathrm{~g}$ i.a./ha, com maior periodo residual para este último. Também trabalhando com abamectin sem fracionamento, com infestações naturais de ácaro rajado Rangel et al. (1990) e Swart et al. (1990) obtiveram bons resultados nas dosagens de 9 e 7,2 g i.a./ha, respectivamente, embora ambos afirmem o baixo efeito de choque e período residual de cerca de 12 dias, aumentado para praticamente 40 dias com a metodologia de vacina,descrita no presente trabalho.

Na Tabela 3 pode ser observado que o abamectin em 3 vezes de $3,6 \mathrm{~g}$ i.a/ha ou 2 vezes de $5,4 \mathrm{~g}$ i.a/ha (efeito vacina) adicionado ao cloreto de mepiquat a $15 \mathrm{~g}$ i.a./ha em 3 vezes, melhorou o porte da planta, reduzindo a níveis inferiores ao uso do cloreto de mepiquat isolado a $15 \mathrm{~g}$ i.a./ha em 3 vezes, embora não a níveis significativos. Observou-se que em ambas as avaliações os tratamentos contendo cloreto de mepiquat a $15 \mathrm{~g}$ i.a./ha em 3 vezes, a altura das plantas foi significativamente menor que nos demais tratamentos, semelhante aos resultados obtidos por Athayde \& Lamas (1999) e Lamas et al. (1999).

Um acréscimo porcentual na produção foi obtido com o uso de todos os tratamentos em relação à testemunha, embora estatisticamente não significante, com ênfase para os tratamentos contendo abamectin, destacando-se o abamectin em 3 vezes de $3,6 \mathrm{~g}$ i.a/ha ou 2 vezes de $5,4 \mathrm{~g}$ i.a/ha (efeito vacina), adicionado ao cloreto de mepiquat a $15 \mathrm{~g}$ i.a./ha em 3 vezes. Lamas (2001) também obteve acréscimos na produção de 
algodão cv. CNPA ITA 90 com o uso de cloreto de mepiquat. Prasad et al. (2000) obtiveram acréscimos na produção utilizando cloreto de mepiquat a $50 \mathrm{~g}$ i.a./ha, em uma única aplicação.

\section{CONCLUSÕES}

Os resultados nos permitem concluir que as aplicações parceladas de abamectin em 3 vezes de $3,6 \mathrm{~g}$ i.a/ha ou 2 vezes de $5,4 \mathrm{~g} \mathrm{i.a/ha}$ (efeito vacina), adicionado ao cloreto de mepiquat a $15 \mathrm{~g}$ i.a./ha em 3 vezes, o ácaro rajado não se desenvolveu, o porte da planta permaneceu adequado à colheita mecanizada. A produção de algodão em caroço foi aumentada, mas não comprovada pela análise estatística.

\section{REFERÊNCIAS BIBLIOGRÁFICAS}

ATHAYDE, M.L.F. \& LAMAS, F.M., 1999. Aplicação Seqüencial de Cloreto de Mepiquat em Algodoeiro. PAB, 34:3:369-375.

ABBOTT, W.S., 1925. A Method of Computing the Effectiveness of on Inseticide, J. Econ. Entomol., 18:255-257.

CALCAGNOLO, G., 1959. Os Laranjais Paulistas estão Prejudicados pelo Ataque de uma Espécie de Ácaro. O Biológico, 25(2):33-38. CHIAVEGATO, L.G., 1971. Flutuação de Populações de Ácaros na Cultura Algodoeira em Algumas Regiões do Estado de São Paulo. Bragantia, 34(15):241-255.

GALLO, D.; NAKANO, O.; SILVEIRA NETO, S.; CARVALHO, R.P.L.;

BATISTA, G.C. de; PARRA, J.R.P.; BERTI FILHO, E.; ZUCCHI, R.A.; ALVES, S.B.; VENDRAMINI, J.D., 1988. Manual de Entomologia Agrícola. Ed. Agronômica Ceres - São Paulo, 531p. GAVIOL,I, L.A.; GRAVENA, S.; LEAO NETO, R.R.; TOZATTI, G., 1987. Efeito de Abamectin, Cyfluthrin e Fenpropathrin sobre os Ácaros Polyphagotarsonemus latus (Banks, 1904) e Tetranychus urticae Koch, 1836), e Alguns Inimigos Naturais no Algodoeiro. Ecossistema, 12:66-67.

LAMAS, F.M.; ATHAYDE, M.L.F.; BANZATTO, D.A.; FORTUNA, P.A., 2001. Cloreto de Mepiquat, Thidiazuron e Ethephon Aplica- 
dos no Algodoeiro em Ponta-Porã, MS. PAB, 34:10:1871-1880. LAMAS, F.M., 2001. Estudo Comparativo entre o Cloreto de Mepiquat

e Cloreto de Chlormequat Aplicados em Algodoeiro. PAB, 36(2):265-273.

NAKANO, O.; SILVEIRA NETO, S. ZUCCHI, R. A., 1981.

Entomologia Econômica. São Paulo; ed. Livroceres, 314p. OLIVEIRA, C.A.L., 1971. Estudos dos Prejuizos Quantitativos e Qualitativos Determinados pela Infestação do Ácaro Rajado Tetranychus urticae, na Cultura Algodoeira. O Biológico, 37(12):341-342. PRASAD, M.; PRAKASH, R., 2000. Influence of Mepiquat on Growth, Yield and Quality of Cotton. Pesticide Res. J., 12(2):261-262. RANGEL, M.C.; REGITANO, E.B.; CLARI, A.I.; HAMAMURA, R.; ARASHIRO, F.Y., RANGEL, R.C.; MARICONI, F.A.M., 1990. Combate Experimental ao Ácaro Rajado do Algodoeiro Tetranychus urticae, 1836 (Acari: Tetranychidae). Anais da Soc. Entomol. do Brasil, 19(1):171-179.

REIS, P.R. 1972. Efeito do Ácaro Tetranychus urticae Koch, 1836 (Acarina: Tetranychidae) na Produção e Qualidade de Fibra do Algodoeiro var. IAC-RM3. Piracicaba 76 p. Dissertação (Mestrado) Escola Superior de Agricultura "Luiz de Queiroz", Universidade de São Paulo.

SWART, M.; PASINI, M.R.; CINIGLIO-NETO, F.; RANGEL, R.C., 1990. Combate ao Ácaro Rajado Tetranychus urticae, 1836 em Algodão com Defensivos Químicos. Anais da Escola Superior de Agricultura Luiz de Queiroz, 47(2):273-282. 